\title{
JOINT DENSITIES OF FIRST HITTING TIMES OF A DIFFUSION PROCESS THROUGH TWO TIME-DEPENDENT BOUNDARIES
}

\author{
LAURA SACERDOTE, ${ }^{* * *}$ \\ OTTAVIA TELVE* AND \\ CRISTINA ZUCCA, ${ }^{*}$ University of Torino
}

\begin{abstract}
Consider a one-dimensional diffusion process on the diffusion interval $I$ originated in $x_{0} \in I$. Let $a(t)$ and $b(t)$ be two continuous functions of $t, t>t_{0}$, with bounded derivatives, $a(t)<b(t)$, and $a(t), b(t) \in I$, for all $t>t_{0}$. We study the joint distribution of the two random variables $T_{a}$ and $T_{b}$, the first hitting times of the diffusion process through the two boundaries $a(t)$ and $b(t)$, respectively. We express the joint distribution of $T_{a}$ and $T_{b}$ in terms of $\mathbb{P}\left(T_{a}<t, T_{a}<T_{b}\right)$ and $\mathbb{P}\left(T_{b}<t, T_{a}>T_{b}\right)$, and we determine a system of integral equations verified by these last probabilities. We propose a numerical algorithm to solve this system and we prove its convergence properties. Examples and modeling motivation for this study are also discussed.
\end{abstract}

Keywords: First hitting time; diffusion process; Brownian motion; Ornstein-Uhlenbeck process; copula

2010 Mathematics Subject Classification: Primary 60J60; 60G40

Secondary 60J70; 65R20

\section{Introduction}

The exit times of diffusion processes from a strip play an important role in a variety of applications ranging from computer science to engineering, from biology to metrology or finance (cf. [2], [9], [21], [23], and [33]). According to the features of the model, constant or time-dependent thresholds may bound the considered process. Typical examples are quality models with two tolerance bands. Some parameter may control exit times from the strip, with different effects on the exit time from the upper or lower bound. The knowledge of the joint exit times probability density function clarifies the role of these parameters. Another example is given by the survival probability of a population in a finite capacity environment or by tumor growth models (cf. [2]). Similar problems arise in metrology when we need to maintain the atomic clock error bounded by two tolerance bands. Moreover, avoiding an excessive increase of the error is of primary importance to improve GPS instruments (cf. [11]). In this setting the knowledge of the relationship between exit times from the upper and the lower boundaries may suggest improvements to the clock reliability by acting on some parameters of the model involved in the joint distribution. Other possible applications can be found in finance where the interest focuses on the dependency between the times to sell or buy options when the level of gain or loss is preassigned. A large body of literature exists for the study of the first passage

Received 17 May 2012; revision received 30 April 2013.

* Postal address: Department of Mathematics 'G. Peano', University of Torino, Via Carlo Alberto 10, 10123 Torino, Italy.

** Email address: laura.sacerdote@unito.it 
time of one-dimensional diffusion processes through a boundary, and analytical, numerical, and simulation methods have been studied both for the direct problem (cf. [4], [6], [14], [15], [24], [27], [30], and [31]) and the inverse problem (cf. [35]). However, most of these papers focus on the one-boundary problem, while, for the two-boundary case, the few analytical results published rely either on the Brownian motion (cf. [22]) or particular time-dependent boundaries, corresponding to special symmetries, for specific diffusions (cf. [7] and [10]). The existing results generally focus on the first exit time from the strip, while our interest lies in the joint distribution of the times when the process first attains the upper and lower boundaries. This paper aims to cover this subject considering the joint distribution between these times. Some results, presented in a recent paper by Giorno et al. [12], are related with those on the Laplace transforms presented in this paper. However, their focus was not on the joint distribution of exit times from a strip.

The notation and the existing results that will be used in this paper are introduced in Section 2, while Sections 3 and 4 are devoted to the presentation of our results. In Section 3 we determine the expression of the joint distribution of the exit times from the upper and lower boundaries. The results are expressed in terms of first hitting time through a single boundary and of the probability of crossing the upper (lower) boundary for the first time at some instant preceding $t$ before crossing the lower (upper) boundary. Note that these probabilities are generally unknown. We prove then that they are the unique solution of a system of Volterra integral equations of the first kind. We also show that there exists an equivalent system of Volterra equations of the second type. When the boundaries are constant, the Laplace transform method can be applied to solve the system, since the integrals of such a system are of convolution type. Here we introduce three equivalent representations of the Laplace transform. In the case of the Brownian motion and constant boundaries, a closed-form expression for the joint distribution of the exit times from a strip is known (cf. [5]).

In Section 4 we propose a numerical scheme for the solution of the system of integral equations and we determine the order of convergence. This method works for both constant and time-dependent boundaries. In the case of two constant boundaries the Laplace transforms (cf. [1]) of the probability of crossing the upper (lower) boundary for the first time at some instant preceding $t$ before crossing the lower (upper) boundary can be numerically inverted. Finally, in Section 5 we present a set of examples.

\section{Mathematical background and notation}

Let $X=\left\{X(t), t \geq t_{0}\right\}$ be a one-dimensional regular time homogeneous diffusion process defined on a suitable probability space $(\Omega, \mathcal{A}, \mathbb{P})$ such that $\mathbb{P}\left(X\left(t_{0}\right)=x_{0}\right)=1$ and with diffusion interval $I$, where $I$ is an interval of the form $\left(r_{1}, r_{2}\right),\left(r_{1}, r_{2}\right],\left[r_{1}, r_{2}\right)$, or $\left[r_{1}, r_{2}\right]$, where $r_{1}=-\infty$ and/or $r_{2}=+\infty$ are admissible when the diffusion interval is open. If not specified, the diffusion interval is open and the endpoints $r_{1}$ and $r_{2}$ are natural boundaries. Let $F_{X(t)}(x \mid y, \tau)=\mathbb{P}(X(t) \leq x \mid X(\tau)=y)$ be the transition probability distribution function (PDF) of the process $X$ and let $f_{X(t)}(x \mid y, \tau)$ be the corresponding transition probability density function.

Let $a(t)$ be a continuous function with bounded derivatives. We denote by $T_{a}$ the first hitting time of the stochastic process $X$ across a boundary $a(t) \in I$ :

$$
T_{a}=\inf \left\{t \geq t_{0}, X(t)=a(t)\right\} .
$$

Its PDF is

$$
F_{T_{a}}\left(t \mid x_{0}, t_{0}\right)=\mathbb{P}\left(T_{a} \leq t \mid X\left(t_{0}\right)=x_{0}\right)
$$


and $f_{T_{a}}\left(t \mid x_{0}, t_{0}\right)$ is the corresponding probability density function. In the case of two boundaries $a(t)<b(t)$ for all $t$, we indicate with $T_{a}$ and $T_{b}$ the first hitting times of the stochastic process $X$ across the boundaries $a(t)$ and $b(t)$, respectively. The aim of this paper is to study the dependency properties of $\left(T_{a}, T_{b}\right)$, i.e. to determine

$$
F_{T_{a}, T_{b}}\left(t, s \mid x_{0}, t_{0}\right)=\mathbb{P}\left(T_{a} \leq t,<T_{b} \leq s \mid X\left(t_{0}\right)=x_{0}\right),
$$

the joint PDF of $\left(T_{a}, T_{b}\right)$, and the corresponding joint probability density function $f_{T_{a}, T_{b}}(t$, $\left.s \mid x_{0}, t_{0}\right)$.

We define the following densities that distinguish the first boundary reached between the two delimiting the strip:

$$
g_{a}\left(t \mid x_{0}, t_{0}\right) \mathrm{d} t=\mathbb{P}\left(T_{a} \in \mathrm{d} t, T_{a}<T_{b} \mid X\left(t_{0}\right)=x_{0}\right)
$$

and

$$
g_{b}\left(t \mid x_{0}, t_{0}\right) \mathrm{d} t=\mathbb{P}\left(T_{b} \in \mathrm{d} t, T_{a}>T_{b} \mid X\left(t_{0}\right)=x_{0}\right) .
$$

For a standard Brownian motion $W=\left\{W(t), t \geq t_{0}\right\}$, the two densities $g_{a}\left(t \mid x_{0}, t_{0}\right)$ and $g_{b}\left(t \mid x_{0}, t_{0}\right)$ are known in closed form (cf. [5]) when the boundaries are constant, $a(t)=a$ and $b(t)=b$ :

$$
\begin{aligned}
& g_{a}\left(t \mid x_{0}, t_{0}\right)=\sum_{k=-\infty}^{\infty} \frac{x_{0}-a+2 k(b-a)}{\sqrt{2 \pi\left(t-t_{0}\right)^{3}}} \exp \left(-\frac{\left(x_{0}-a+2 k(b-a)\right)^{2}}{2\left(t-t_{0}\right)}\right), \\
& g_{b}\left(t \mid x_{0}, t_{0}\right)=\sum_{k=-\infty}^{\infty} \frac{b-x_{0}+2 k(b-a)}{\sqrt{2 \pi\left(t-t_{0}\right)^{3}}} \exp \left(-\frac{\left(b-x_{0}+2 k(b-a)\right)^{2}}{2\left(t-t_{0}\right)}\right) .
\end{aligned}
$$

The probability density function and PDF of $T_{a}$ are

$$
\begin{gathered}
f_{T_{a}}\left(t \mid x_{0}, t_{0}\right)=\frac{\left|a-x_{0}\right|}{\sqrt{2 \pi\left(t-t_{0}\right)^{3}}} \exp \left(-\frac{\left(a-x_{0}\right)^{2}}{2\left(t-t_{0}\right)}\right), \\
F_{T_{a}}\left(t \mid x_{0}, t_{0}\right)=1-\operatorname{erf}\left(\frac{\left|a-x_{0}\right|}{\sqrt{2 t}}\right) .
\end{gathered}
$$

Quantities (1) and (2) are useful for the computation of the joint density function of $T_{a}$ and $T_{b}$. Two different instances arise according to the location of the starting point $X\left(t_{0}\right)=x_{0}$ with respect to the boundaries (cf. Figure 1). The following result holds.

Theorem 1. Let $X=\left\{X(t), t \geq t_{0}\right\}$ be a diffusion process such that $X\left(t_{0}\right)=x_{0}$, and let $a(t)$ and $b(t)$ be two continuous time-dependent boundaries.

(i) If $x_{0}<a\left(t_{0}\right)<b\left(t_{0}\right)$ and $a(t)<b(t)$ for each $t>t_{0}$ or $b\left(t_{0}\right)<a\left(t_{0}\right)<x_{0}$ and $b(t)<a(t)$ for each $t>t_{0}$, then

$$
f_{T_{a}, T_{b}}\left(t, s \mid x_{0}, t_{0}\right)= \begin{cases}0, & t \geq s, \\ f_{T_{a}}\left(t \mid x_{0}, t_{0}\right) f_{T_{b}}(s \mid a(t), t), & t<s .\end{cases}
$$

(ii) If $a\left(t_{0}\right)<x_{0}<b\left(t_{0}\right)$ and $a(t)<b(t)$ for each $t>t_{0}$, then

$$
f_{T_{a} T_{b}}\left(t, s \mid x_{0}, t_{0}\right)= \begin{cases}f_{T_{b}}(s \mid a(t), t) g_{a}\left(t \mid x_{0}, t_{0}\right), & t<s, \\ 0, & t=s, \\ f_{T_{a}}(t \mid b(s), s) g_{b}\left(s \mid x_{0}, t_{0}\right), & t>s .\end{cases}
$$

We omit the proof as it straightforwardly follows using the strong Markov property. 
(a)

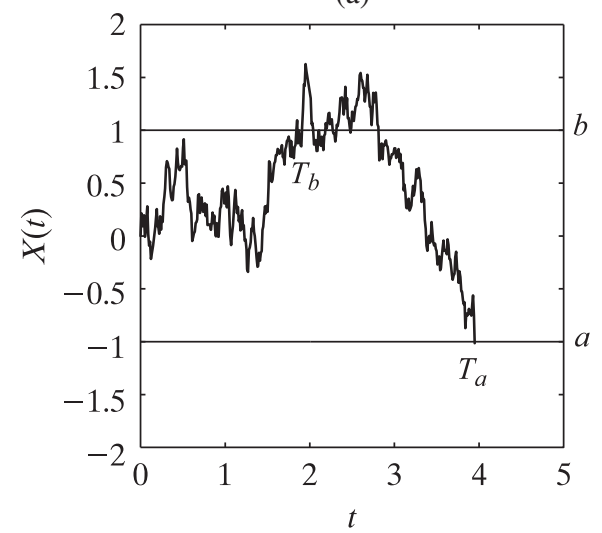

(b)

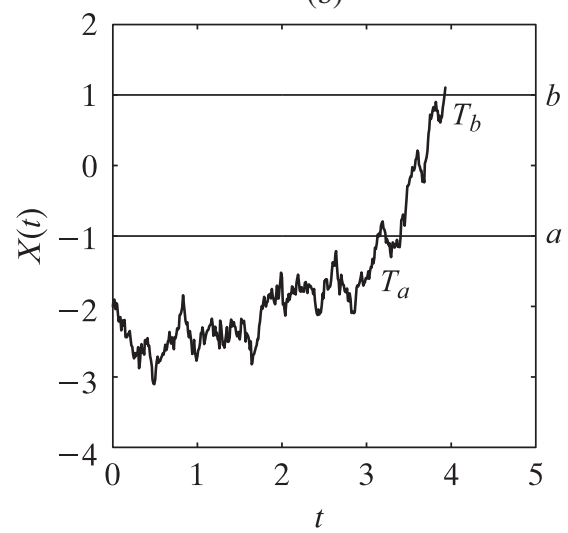

FIGURE 1: Sample path of a stochastic process through two constant boundaries $a$ and $b$. (a) $x_{0} \in(a, b)$. (b) $x_{0} \notin(a, b)$.

Remark 1. Note that the first hitting time probability density function verifies the initial condition

$$
\lim _{s \rightarrow t} f_{T_{b}}(s \mid a(t), t)=\lim _{t \rightarrow s} f_{T_{a}}(t \mid b(s), s)=0 .
$$

Furthermore, due to the differentiability of the boundaries, it holds (cf. [29]) that

$$
\begin{gathered}
\lim _{t \rightarrow s} F_{X(t)}(b(t) \mid b(s), s)=\lim _{t \rightarrow s} F_{X(t)}(a(t) \mid a(s), s)=\frac{1}{2}, \\
\lim _{t \rightarrow s}\left[1-F_{X(t)}(b(t) \mid a(s), s)\right]=\lim _{t \rightarrow s} F_{X(t)}(a(t) \mid b(s), s)=0 .
\end{gathered}
$$

Remark 2. For some applications, it might be interesting to determine the copula function (cf. [20]) between $T_{a}$ and $T_{b}$. When the two densities $g_{a}\left(t \mid x_{0}, t_{0}\right)$ and $g_{b}\left(t \mid x_{0}, t_{0}\right)$ are known, use of (5) and (6) allows us to determine this function.

\section{System of integral equations}

The computation of $f_{T_{a} T_{b}}\left(t, s \mid x_{0}, t_{0}\right)$ involves the transition probability density functions $f_{T_{b}}(s \mid a(t), t)$ and $f_{T_{a}}(t \mid b(s), s)$ and the terms $g_{a}\left(t \mid x_{0}, t_{0}\right)$ and $g_{b}\left(t \mid x_{0}, t_{0}\right)$. When the process is a linear regular diffusion, the transition probability density function is available in closed form and, if the process is strictly linear, it is Gaussian. In the literature, the transition probability density function is also available for other regular diffusion processes, such as the Cox-Ingersoll-Ross model (also known as the Feller process), the Bessel process, or some instances of the Raleigh process (cf. [13] and [14]). Further examples arise from the spacetime transformation of the Brownian motion (cf. [25]) or of the Cox-Ingersoll-Ross process (cf. [8]). When closed-form solutions are not available, the transition probability density function is evaluated via numerical methods, such as the numerical solution of the Kolmogorov equation [17], [32] or the numerical inversion of Fourier transforms [34]. Unfortunately, closed-form expressions for the densities $g_{a}\left(t \mid x_{0}, t_{0}\right)$ and $g_{b}\left(t \mid x_{0}, t_{0}\right)$ are known only for the Brownian motion with constant boundaries (cf. [5, Formula 3.0.6]) or processes related to it through suitable transformations. Use of the following theorem helps to overcome this problem.

Theorem 2. Let $X=\left\{X(t), t \geq t_{0}\right\}$ be a diffusion process such that $X\left(t_{0}\right)=x_{0}$. Let $a(t)$ and $b(t)$ be two time-dependent boundaries with bounded derivatives such that $a\left(t_{0}\right)<x_{0}<$ $b\left(t_{0}\right)$ and $a(t)<b(t)$ for each $t>t_{0}$. The probability density functions $g_{a}\left(t \mid x_{0}, t_{0}\right)$ and 
$g_{b}\left(t \mid x_{0}, t_{0}\right)$ are solutions of the following system of Volterra first-kind integral equations:

$$
\begin{aligned}
1-F_{X(t)}\left(b(t) \mid x_{0}, t_{0}\right)= & \int_{t_{0}}^{t}\left[1-F_{X(t)}(b(t) \mid a(\tau), \tau)\right] g_{a}\left(\tau \mid x_{0}, t_{0}\right) \mathrm{d} \tau \\
& +\int_{t_{0}}^{t}\left[1-F_{X(t)}(b(t) \mid b(\tau), \tau)\right] g_{b}\left(\tau \mid x_{0}, t_{0}\right) \mathrm{d} \tau, \\
F_{X(t)}\left(a(t) \mid x_{0}, t_{0}\right)= & \int_{t_{0}}^{t} F_{X(t)}(a(t) \mid a(\tau), \tau) g_{a}\left(\tau \mid x_{0}, t_{0}\right) \mathrm{d} \tau \\
& +\int_{t_{0}}^{t} F_{X(t)}(a(t) \mid b(\tau), \tau) g_{b}\left(\tau \mid x_{0}, t_{0}\right) \mathrm{d} \tau
\end{aligned}
$$

Proof. For $t \in[0, \infty]$, conditioning on the boundary first attained by the process, for $x \notin[a(t), b(t)]$, we obtain

$$
\begin{aligned}
\mathbb{P}(X(t) & \left.\leq x \mid X\left(t_{0}\right)=x_{0}\right) \\
= & \int_{t_{0}}^{t} \mathbb{P}\left(X(t) \leq x \mid T_{a}<T_{b}, T_{a}=\tau, X\left(t_{0}\right)=x_{0}\right) g_{a}\left(\tau \mid x_{0}, t_{0}\right) \mathrm{d} \tau \\
& +\int_{t_{0}}^{t} \mathbb{P}\left(X(t) \leq x \mid T_{a}>T_{b}, T_{b}=\tau, X\left(t_{0}\right)=x_{0}\right) g_{b}\left(\tau \mid x_{0}, t_{0}\right) \mathrm{d} \tau \\
= & \int_{t_{0}}^{t} \mathbb{P}\left(X(t) \leq x \mid X(\tau)=a(\tau), X\left(t_{0}\right)=x_{0}\right) g_{a}\left(\tau \mid x_{0}, t_{0}\right) \mathrm{d} \tau \\
& +\int_{t_{0}}^{t} \mathbb{P}\left(X(t) \leq x \mid X(\tau)=b(\tau), X\left(t_{0}\right)=x_{0}\right) g_{b}\left(\tau \mid x_{0}, t_{0}\right) \mathrm{d} \tau .
\end{aligned}
$$

Differentiating with respect to $x$ we obtain

$$
\begin{aligned}
f_{X(t)}\left(x \mid x_{0}, t_{0}\right)= & \int_{t_{0}}^{t} f_{X(t)}(x \mid a(\tau), \tau) g_{a}\left(\tau \mid x_{0}, t_{0}\right) \mathrm{d} \tau \\
& +\int_{t_{0}}^{t} f_{X(t)}(x \mid b(\tau), \tau) g_{b}\left(\tau \mid x_{0}, t_{0}\right) \mathrm{d} \tau
\end{aligned}
$$

Integrating (10) with respect to $x$ on the two subdomains $[b(t), \infty]$ and $[-\infty, a(t)]$, we respectively obtain $(9 a)$ and $(9 b)$.

Remark 3. Differentiating (9a) and (9b) with respect to $t$, and recalling (7) and (8), we obtain

$$
\begin{aligned}
g_{b}\left(t \mid x_{0}, t_{0}\right)= & -2 \frac{\partial F_{X(t)}\left(b(t) \mid x_{0}, t_{0}\right)}{\partial t} \\
& +\int_{t_{0}}^{t} 2\left(\frac{\partial F_{X(t)}(b(t) \mid a(\tau), \tau)}{\partial t} g_{a}\left(\tau \mid x_{0}, t_{0}\right)\right. \\
& \left.+\frac{\partial F_{X(t)}(b(t) \mid b(\tau), \tau)}{\partial t} g_{b}\left(\tau \mid x_{0}, t_{0}\right)\right) \mathrm{d} \tau \\
g_{a}\left(t \mid x_{0}, t_{0}\right)= & 2 \frac{\partial F_{X(t)}\left(a(t) \mid x_{0}, t_{0}\right)}{\partial t} \\
& -\int_{t_{0}}^{t} 2\left(\frac{\partial F_{X(t)}(a(t) \mid a(\tau), \tau)}{\partial t} g_{a}\left(\tau \mid x_{0}, t_{0}\right)\right. \\
& \left.+\frac{\partial F_{X(t)}(a(t) \mid b(\tau), \tau)}{\partial t} g_{b}\left(\tau \mid x_{0}, t_{0}\right)\right) \mathrm{d} \tau .
\end{aligned}
$$


This system of Volterra integral equations coincides with that proposed in [7] if the kernel of the two equations is regularized.

The following result holds.

Theorem 3. System (9) has a unique continuous solution for $t>t_{0}$.

Proof. The system of Volterra integral equations of the first kind, (9), is equivalent to the system of Volterra integral equations of the second kind, (11), that can be written in matricial form

$$
\boldsymbol{g}(t)=\boldsymbol{h}(t)+\int_{t_{0}}^{t} \boldsymbol{k}(t, \tau) \boldsymbol{g}(\tau) \mathrm{d} \tau,
$$

where

$$
\begin{gathered}
\boldsymbol{g}(t)=\left[\begin{array}{l}
g_{a}\left(t \mid x_{0}, t_{0}\right) \\
g_{b}\left(t \mid x_{0}, t_{0}\right)
\end{array}\right], \quad \boldsymbol{h}(t)=\left[\begin{array}{c}
-2 \frac{\partial F_{X(t)}\left(b(t) \mid x_{0}, t_{0}\right)}{\partial t} \\
2 \frac{\partial F_{X(t)}\left(a(t) \mid x_{0}, t_{0}\right)}{\partial t}
\end{array}\right], \\
\boldsymbol{k}(t, \tau)=\left[\begin{array}{ll}
-2 \frac{\partial F_{X(t)}(b(t) \mid a(\tau), \tau)}{\partial t} & -2 \frac{\partial F_{X(t)}(b(t) \mid b(\tau), \tau)}{\partial t} \\
2 \frac{\partial F_{X(t)}(a(t) \mid a(\tau), \tau)}{\partial t} & 2 \frac{\partial F_{X(t)}(a(t) \mid b(\tau), \tau)}{\partial t}
\end{array}\right] .
\end{gathered}
$$

Since the kernel $\boldsymbol{k}(t, \tau)$ is singular in $\tau=t$, we introduce an equivalent system with continuous kernel. Mimicking the method presented in [6], we introduce the functions $\gamma_{i}(t)$ and $\eta_{i}(t), i=$ 1,2 , continuous in $\left[t_{0},+\infty\right]$. Combining (9), (10), and (11), together with $\gamma_{i}(t)$ and $\eta_{i}(t)$, we obtain

$$
\begin{aligned}
g_{b}\left(t \mid x_{0}, t_{0}\right)=-\Psi^{1}\left(b(t) \mid x_{0}, t_{0}\right)+\int_{t_{0}}^{t} & \left(\Psi^{1}(b(t) \mid a(\tau), \tau) g_{a}\left(\tau \mid x_{0}, t_{0}\right)\right. \\
& \left.+\Psi^{1}(b(t) \mid b(\tau), \tau) g_{b}\left(\tau \mid x_{0}, t_{0}\right)\right) \mathrm{d} \tau \\
g_{a}\left(t \mid x_{0}, t_{0}\right)=\Psi^{2}\left(a(t) \mid x_{0}, t_{0}\right)-\int_{t_{0}}^{t}( & \Psi^{2}(a(t) \mid a(\tau), \tau) g_{a}\left(\tau \mid x_{0}, t_{0}\right) \\
& \left.+\Psi^{2}(a(t) \mid b(\tau), \tau) g_{b}\left(\tau \mid x_{0}, t_{0}\right)\right) \mathrm{d} \tau
\end{aligned}
$$

where

$$
\begin{aligned}
\Psi^{1}(b(t) \mid x, s)= & -2 \frac{\partial F_{X(t)}(b(t) \mid x, s)}{\partial t}+\gamma_{1}(t) f_{X(t)}(b(t), t \mid x, s) \\
& +\eta_{1}(t)\left[1-F_{X(t)}(b(t) \mid x, s)\right], \\
\Psi^{2}(a(t) \mid x, s)= & 2 \frac{\partial F_{X(t)}(a(t) \mid x, s)}{\partial t}-\gamma_{2}(t) f_{X(t)}(a(t), t \mid x, s)-\eta_{2}(t) F_{X(t)}(a(t) \mid x, s) .
\end{aligned}
$$

A suitable choice of $\gamma_{i}(t)$ and $\eta_{i}(t), i=1,2$, makes both $\Psi^{1}(b(t) \mid b(\tau), \tau)$ and $\Psi^{2}(a(t) \mid a(\tau), \tau)$ not singular. On the other hand, since (cf. [7])

$$
\begin{aligned}
& \lim _{\tau \rightarrow t} f_{X(t)}(b(t), t \mid a(\tau), \tau)=\left.\lim _{\tau \rightarrow t} \frac{\partial}{\partial x} f_{X(t)}(x, t \mid a(\tau), \tau)\right|_{x=b(t)}=0, \\
& \lim _{\tau \rightarrow t} f_{X(t)}(a(t), t \mid b(\tau), \tau)=\left.\lim _{\tau \rightarrow t} \frac{\partial}{\partial x} f_{X(t)}(x, t \mid b(\tau), \tau)\right|_{x=a(t)}=0,
\end{aligned}
$$


the kernels $\Psi^{1}(b(t) \mid a(\tau), \tau)$ and $\Psi^{2}(a(t) \mid b(\tau), \tau)$ are not singular. This makes it possible to apply Theorem 3.11 of [19] to obtain the thesis.

Remark 4. The functions $\gamma_{i}(t)$ and $\eta_{i}(t), i=1,2$, can be determined. For example, for an Ornstein-Uhlenbeck process characterized by the drift $\mu(t, x)=\alpha x+\beta$ and infinitesimal variance $\sigma(t, x)=\sigma$, where $\alpha, \beta$, and $\sigma>0$ are arbitrary real constants. The functions that regularize the kernels are $\gamma_{1}(t)=1 / 2\left[\alpha b(t)+\beta-b^{\prime}(t)\right], \gamma_{2}(t)=1 / 2\left[\alpha a(t)+\beta-a^{\prime}(t)\right]$, and $\eta_{i}(t) \equiv 0, i=1,2$ (cf. [6]).

When the boundaries are constant, the following result holds.

Corollary 1. Let $X=\left\{X(t), t \geq t_{0}\right\}$ be a diffusion process such that $X\left(t_{0}\right)=x_{0}$, and let a and $b$ be two constant boundaries such that $a<x_{0}<b$. Then the following three expressions are equivalent for $g_{a}^{\lambda}\left(x_{0}\right)=\int_{t_{0}}^{+\infty} \mathrm{e}^{-\lambda t} g_{a}\left(t \mid x_{0}, t_{0}\right) \mathrm{d} t$ and $g_{b}^{\lambda}\left(x_{0}\right)=\int_{t_{0}}^{+\infty} \mathrm{e}^{-\lambda t} g_{b}\left(t \mid x_{0}, t_{0}\right) \mathrm{d} t$ :

$$
\begin{gathered}
g_{a}^{\lambda}\left(x_{0}\right)=\frac{f_{T_{b}}^{\lambda}\left(x_{0}\right) f_{T_{a}}^{\lambda}(b)-f_{T_{a}}^{\lambda}\left(x_{0}\right)}{f_{T_{a}}^{\lambda}(b) f_{T_{b}}^{\lambda}(a)-1}, \\
g_{b}^{\lambda}\left(x_{0}\right)=\frac{f_{T_{a}}^{\lambda}\left(x_{0}\right) f_{T_{b}}^{\lambda}(a)-f_{T_{b}}^{\lambda}\left(x_{0}\right)}{f_{T_{a}}^{\lambda}(b) f_{T_{b}}^{\lambda}(a)-1}, \\
g_{a}^{\lambda}\left(x_{0}\right)=\frac{\left[1-\lambda F_{X}^{\lambda}\left(b \mid x_{0}\right)\right] F_{X}^{\lambda}(a \mid b)-\left[1-\lambda F_{X}^{\lambda}(b \mid b)\right] F_{X}^{\lambda}\left(a \mid x_{0}\right)}{\left[1-\lambda F_{X}^{\lambda}(b \mid a)\right] F_{X}^{\lambda}(a \mid b)-\left[1-\lambda F_{X}^{\lambda}(b \mid b)\right] F_{X}^{\lambda}(a \mid a)}, \\
g_{b}^{\lambda}\left(x_{0}\right)=\frac{\left[1-\lambda F_{X}^{\lambda}\left(b \mid x_{0}\right)\right] F_{X}^{\lambda}(a \mid a)-\left[1-\lambda F_{X}^{\lambda}(b \mid a)\right] F_{X}^{\lambda}\left(a \mid x_{0}\right)}{\left[1-\lambda F_{X}^{\lambda}(b \mid b)\right] F_{X}^{\lambda}(a \mid a)-\left[1-\lambda F_{X}^{\lambda}(b \mid a)\right] F_{X}^{\lambda}(a \mid b)}, \\
g_{a}^{\lambda}\left(x_{0}\right)=\frac{f_{X}^{\lambda}\left(x_{1} \mid x_{0}\right) f_{X}^{\lambda}\left(x_{2} \mid b\right)-f_{X}^{\lambda}\left(x_{1} \mid b\right) f_{X}^{\lambda}\left(x_{2} \mid x_{0}\right)}{f_{X}^{\lambda}\left(x_{1} \mid a\right) f_{X}^{\lambda}\left(x_{2} \mid b\right)-f_{X}^{\lambda}\left(x_{1} \mid b\right) f_{X}^{\lambda}\left(x_{2} \mid a\right)} \\
=\frac{v_{1}\left(\alpha, x_{0}\right) v_{2}(\alpha, b)-v_{2}\left(\alpha, x_{0}\right) v_{1}(\alpha, b)}{v_{1}(\alpha, a) v_{2}(\alpha, b)-v_{2}(\alpha, a) v_{1}(\alpha, b)}, \\
g_{b}^{\lambda}\left(x_{0}\right)=\frac{f_{X}^{\lambda}\left(x_{1} \mid a\right) f_{X}^{\lambda}\left(x_{2} \mid x_{0}\right)-f_{X}^{\lambda}\left(x_{1} \mid x_{0}\right) f_{X}^{\lambda}\left(x_{2} \mid a\right)}{f_{X}^{\lambda}\left(x_{1} \mid a\right) f_{X}^{\lambda}\left(x_{2} \mid b\right)-f_{X}^{\lambda}\left(x_{1} \mid b\right) f_{X}^{\lambda}\left(x_{2} \mid a\right)} \\
=\frac{v_{1}(\alpha, a) v_{2}\left(\alpha, x_{0}\right)-v_{2}(\alpha, a) v_{1}\left(\alpha, x_{0}\right)}{v_{1}(\alpha, a) v_{2}(\alpha, b)-v_{2}(\alpha, a) v_{1}(\alpha, b)},
\end{gathered}
$$

where

$$
\begin{aligned}
F_{X}^{\lambda}\left(x \mid x_{0}\right) & =\int_{0}^{+\infty} \mathrm{e}^{-\lambda t} F_{X(t)}\left(x \mid x_{0}, t_{0}\right) \mathrm{d} t, \\
f_{X}^{\lambda}\left(x \mid x_{0}\right) & =\int_{0}^{+\infty} \mathrm{e}^{-\lambda t} f_{X(t)}\left(x \mid x_{0}, t_{0}\right) \mathrm{d} t \\
f_{T_{a}}^{\lambda}\left(x_{0}\right) & =\int_{0}^{+\infty} \mathrm{e}^{-\lambda t} F_{T_{a}}\left(t \mid x_{0}, t_{0}\right) \mathrm{d} t
\end{aligned}
$$

and the functions $v_{i}(\alpha, x), i=1,2$, are fundamental solutions of Equation (8.13b) of [28].

Proof. Generalizing the standard calculation given in [16, p. 30] for an arbitrary regular diffusion yields (12). 
Applying the Laplace transform to (9) and using the convolution theorem we obtain (13), a result recently published in [12]. Applying the Laplace transform to (10) together with the convolution theorem for two generic points $x_{1}>b$ and $x_{2}<a$ gives the first equality in (14). The use of Equation (8.22) of [28] allows us to obtain the second equality.

Furthermore, recalling that (cf. [26])

$$
f_{T_{a}}^{\lambda}\left(x_{1} \mid x_{0}\right)=\frac{f_{X}^{\lambda}\left(x \mid x_{0}\right)}{f_{X}^{\lambda}(x \mid a)},
$$

the first equality in (14) becomes (12).

Finally, remembering that (cf. [26])

$$
f_{T_{a}}^{\lambda}\left(x_{1} \mid x_{0}\right)= \begin{cases}\frac{1-\lambda F_{X}^{\lambda}\left(a \mid x_{0}\right)}{1-\lambda F_{X}^{\lambda}(a \mid a)} & \text { if } a>x_{0}, \\ \frac{F_{X}^{\lambda}\left(a \mid x_{0}\right)}{F_{X}^{\lambda}(a \mid a)} & \text { if } a<x_{0},\end{cases}
$$

(12a), (12b) become (13a), (13b). This implies that the three formulations are equivalent.

Remark 5. The above results also hold for diffusion processes bounded by one or two reflecting boundaries when the diffusion interval is characterized by nonnatural boundaries, i.e. for CoxIngersoll-Ross, whose diffusion interval is $I=[0, \infty)$, or for the reflected Brownian motion.

\section{Algorithms for $\mathbb{P}\left(T_{a} \in \mathrm{d} t, T_{a}<T_{b}\right)$ and $\mathbb{P}\left(T_{b} \in \mathrm{d} t, T_{a}>T_{b}\right)$}

In this section we describe two approaches to determine the density functions $g_{a}\left(t \mid x_{0}, t_{0}\right)$ and $g_{b}\left(t \mid x_{0}, t_{0}\right)$.

When the boundaries are constant, the densities $g_{a}\left(t \mid x_{0}, t_{0}\right)$ and $g_{b}\left(t \mid x_{0}, t_{0}\right)$ are obtained from the Laplace transforms (13) by inverting them numerically using, for example, the Euler method [1].

Alternative methods become necessary when the boundaries $a(t)$ and $b(t)$ are time dependent or when the Laplace inversion presents numerical difficulties. For example, in the case of the Ornstein-Uhlenbeck process the expressions of $g_{a}^{\lambda}\left(x_{0}\right)$ and $g_{b}^{\lambda}\left(x_{0}\right)$ involve a parabolic cylinder function (cf. [31]). Their numerical inversion requires effort specific to this instance. Furthermore, there are processes for which $F_{X}^{\lambda}\left(a \mid x_{0}\right)$ and $F_{X}^{\lambda}\left(b \mid x_{0}\right)$ are not known in the literature. Their computation is possible; however, it requires the solution of specific secondorder differential equations (cf. [26]).

Here we propose the following numerical method that can be applied both for constant and time-dependent boundaries. Let us introduce the time discretization $t_{i}=t_{0}+i h, i=1,2, \ldots$, where $h$ is a positive constant. To determine the two probability density functions $g_{a}\left(t \mid x_{0}, t_{0}\right)$ and $g_{b}\left(t \mid x_{0}, t_{0}\right)$ at the finite set of knots $t_{i}$ for $i=1, \ldots, n$, we use the Euler method [19] to approximate the integrals on the right-hand side of (9a) and (9b). Hence, we obtain

$$
\begin{aligned}
1-F_{X\left(t_{i}\right)}\left(b\left(t_{i}\right) \mid x_{0}, t_{0}\right)= & \sum_{j=1}^{i}\left[1-F_{X\left(t_{i}\right)}\left(b\left(t_{i}\right) \mid a\left(t_{j}\right), t_{j}\right)\right] \hat{g}_{a}\left(t_{j} \mid x_{0}, t_{0}\right) h \\
& +\sum_{j=1}^{i}\left[1-F_{X\left(t_{i}\right)}\left(b\left(t_{i}\right) \mid b\left(t_{j}\right), t_{j}\right)\right] \hat{g}_{b}\left(t_{j} \mid x_{0}, t_{0}\right) h,
\end{aligned}
$$




$$
\begin{aligned}
F_{X\left(t_{i}\right)}\left(a\left(t_{i}\right) \mid x_{0}, t_{0}\right)= & \sum_{j=1}^{i} F_{X\left(t_{i}\right)}\left(a\left(t_{i}\right) \mid a\left(t_{j}\right), t_{j}\right) \hat{g}_{a}\left(t_{j} \mid x_{0}, t_{0}\right) h \\
& +\sum_{j=1}^{i} F_{X\left(t_{i}\right)}\left(a\left(t_{i}\right) \mid b\left(t_{j}\right), t_{j}\right) \hat{g}_{b}\left(t_{j} \mid x_{0}, t_{0}\right) h .
\end{aligned}
$$

The densities $g_{a}\left(t \mid x_{0}, t_{0}\right)$ and $g_{b}\left(t \mid x_{0}, t_{0}\right)$ can be evaluated in the knots $t_{i}$ for $i=1, \ldots, n$ by means of the following algorithm.

Step 1. We have

$$
\begin{gathered}
\hat{g}_{b}\left(t_{1} \mid x_{0}, t_{0}\right)=\frac{2\left(1-F_{X\left(t_{1}\right)}\left(b\left(t_{1}\right) \mid x_{0}, t_{0}\right)\right)}{h}, \\
\hat{g}_{a}\left(t_{1} \mid x_{0}, t_{0}\right)=\frac{2 F_{X\left(t_{1}\right)}\left(a\left(t_{1}\right) \mid x_{0}, t_{0}\right)}{h} .
\end{gathered}
$$

Step $i, i=2,3, \ldots$. We have

$$
\begin{aligned}
\hat{g}_{b}\left(t_{i} \mid x_{0}, t_{0}\right)= & \frac{2\left(1-F_{X\left(t_{i}\right)}\left(b\left(t_{i}\right) \mid x_{0}, t_{0}\right)\right)}{h} \\
& -2 \sum_{j=1}^{i-1}\left[\left(1-F_{X\left(t_{i}\right)}\left(b\left(t_{i}\right) \mid a\left(t_{j}\right), t_{j}\right)\right) \hat{g}_{a}\left(t_{j} \mid x_{0}, t_{0}\right)\right. \\
\left.\quad+\left(1-F_{X\left(t_{i}\right)}\left(b\left(t_{i}\right) \mid b\left(t_{j}\right), t_{j}\right)\right) \hat{g}_{b}\left(t_{j} \mid x_{0}, t_{0}\right)\right], & \\
\hat{g}_{a}\left(t_{i} \mid x_{0}, t_{0}\right)= & \frac{2 F_{X\left(t_{i}\right)}\left(a\left(t_{i}\right) \mid x_{0}, t_{0}\right)}{h} \\
& -2 \sum_{j=1}^{i-1}\left[F_{X\left(t_{i}\right)}\left(a\left(t_{i}\right) \mid a\left(t_{j}\right), t_{j}\right) \hat{g}_{a}\left(t_{j} \mid x_{0}, t_{0}\right)\right. \\
& \left.+F_{X\left(t_{i}\right)}\left(a\left(t_{i}\right) \mid b\left(t_{j}\right), t_{j}\right) \hat{g}_{b}\left(t_{j} \mid x_{0}, t_{0}\right)\right],
\end{aligned}
$$

where we have used (7) and (8).

Remark 6. The choice of equally spaced knots is motivated by the simplification of the notation, but the method can be easily extended to nonconstant $h$.

Theorem 4. If constants $c_{1}$ and $c_{2}$ exist such that, for all $h>0$,

$$
\begin{array}{r}
\max _{1<i<n, 0<j \leq i-1}\left|F_{X\left(t_{i}\right)}\left(b\left(t_{i}\right) \mid a\left(t_{j}\right), t_{j}\right)-F_{X\left(t_{i-1}\right)}\left(b\left(t_{i-1}\right) \mid a\left(t_{j}\right), t_{j}\right)\right| \leq c_{1} h, \\
\max _{1<i<n, 0<j \leq i-1}\left|F_{X\left(t_{i}\right)}\left(a\left(t_{i}\right) \mid b\left(t_{j}\right), t_{j}\right)-F_{X\left(t_{i-1}\right)}\left(a\left(t_{i-1}\right) \mid b\left(t_{j}\right), t_{j}\right)\right| \leq c_{2} h
\end{array}
$$

then the absolute value of the errors $\varepsilon_{a, i}$ and $\varepsilon_{b, i}$ of the proposed algorithm at the discretization knots $t_{i}, i=1,2, \ldots$,

$$
\begin{aligned}
\varepsilon_{a, i}:=\hat{g}_{a}\left(t_{i} \mid x_{0}, t_{0}\right)-g_{a}\left(t_{i} \mid x_{0}, t_{0}\right), \\
\varepsilon_{b, i}:=\hat{g}_{b}\left(t_{i} \mid x_{0}, t_{0}\right)-g_{b}\left(t_{i} \mid x_{0}, t_{0}\right),
\end{aligned}
$$

are $O(h)$. 
Proof. The Euler method applied to (9) gives

$$
\begin{aligned}
1-F_{X\left(t_{i}\right)}\left(b\left(t_{i}\right) \mid x_{0}, t_{0}\right)= & \sum_{j=1}^{i}\left[1-F_{X\left(t_{i}\right)}\left(b\left(t_{i}\right) \mid a\left(t_{j}\right), t_{j}\right)\right] g_{a}\left(t_{j} \mid x_{0}, t_{0}\right) h \\
& +\sum_{j=1}^{i}\left[1-F_{X\left(t_{i}\right)}\left(b\left(t_{i}\right) \mid b\left(t_{j}\right), t_{j}\right)\right] g_{b}\left(t_{j} \mid x_{0}, t_{0}\right) h, \\
& +\delta_{1}\left(h, t_{n}\right) \\
F_{X\left(t_{i}\right)}\left(a\left(t_{i}\right) \mid x_{0}, t_{0}\right)= & \sum_{j=1}^{i} F_{X\left(t_{i}\right)}\left(a\left(t_{i}\right) \mid a\left(t_{j}\right), t_{j}\right) g_{a}\left(t_{j} \mid x_{0}, t_{0}\right) h \\
& +\sum_{j=1}^{i} F_{X\left(t_{i}\right)}\left(a\left(t_{i}\right) \mid b\left(t_{j}\right), t_{j}\right) g_{b}\left(t_{j} \mid x_{0}, t_{0}\right) h+\delta_{2}\left(h, t_{n}\right),
\end{aligned}
$$

where $\delta_{1}\left(h, t_{i}\right)$ and $\delta_{2}\left(h, t_{i}\right)$ are the differences between the integrals on the right-hand side of (9) and the finite sums on the right-hand side of (18).

On subtracting (18a) from (15a) and (18b) from (15b) we obtain

$$
\begin{aligned}
\delta_{1}\left(h, t_{i}\right)= & h \sum_{j=1}^{i}\left[1-F_{X\left(t_{i}\right)}\left(b\left(t_{i}\right) \mid a\left(t_{j}\right), t_{j}\right)\right] \varepsilon_{a, j} \\
& +h \sum_{j=1}^{i}\left[1-F_{X\left(t_{i}\right)}\left(b\left(t_{i}\right) \mid b\left(t_{j}\right), t_{j}\right)\right] \varepsilon_{b, j}, \\
\delta_{2}\left(h, t_{i}\right)= & h \sum_{j=1}^{i} F_{X\left(t_{i}\right)}\left(a\left(t_{i}\right) \mid a\left(t_{j}\right), t_{j}\right) \varepsilon_{a, j}+h \sum_{j=1}^{i} F_{X\left(t_{i}\right)}\left(a\left(t_{i}\right) \mid b\left(t_{j}\right), t_{j}\right) \varepsilon_{b, j} .
\end{aligned}
$$

Differencing (19) and recalling (7) and (8), we obtain

$$
\begin{aligned}
\delta_{1}\left(h, t_{i}\right) & -\delta_{1}\left(h, t_{i-1}\right) \\
= & \frac{h}{2} \varepsilon_{b, i}+h \sum_{j=1}^{i-1}\left(\left[1-F_{X\left(t_{i}\right)}\left(b\left(t_{i}\right) \mid a\left(t_{j}\right), t_{j}\right)\right]-\left[1-F_{X\left(t_{i-1}\right)}\left(b\left(t_{i-1}\right) \mid a\left(t_{j}\right), t_{j}\right)\right]\right) \varepsilon_{a, j} \\
& +h \sum_{j=1}^{i-1}\left(\left[1-F_{X\left(t_{i}\right)}\left(b\left(t_{i}\right) \mid b\left(t_{j}\right), t_{j}\right)\right]-\left[1-F_{X\left(t_{i-1}\right)}\left(b\left(t_{i-1}\right) \mid b\left(t_{j}\right), t_{j}\right)\right]\right) \varepsilon_{b, j}, \\
\delta_{2}\left(h, t_{i}\right) & -\delta_{2}\left(h, t_{i-1}\right) \\
= & \frac{h}{2} \varepsilon_{a, i}+h \sum_{j=1}^{i-1}\left(F_{X\left(t_{i}\right)}\left(a\left(t_{i}\right) \mid a\left(t_{j}\right), t_{j}\right)-F_{X\left(t_{i-1}\right)}\left(a\left(t_{i-1}\right) \mid a\left(t_{j}\right), t_{j}\right)\right) \varepsilon_{a, j} \\
& +\sum_{j=1}^{i-1}\left(F_{X\left(t_{i}\right)}\left(a\left(t_{i}\right) \mid b\left(t_{j}\right), t_{j}\right)-F_{X\left(t_{i-1}\right)}\left(a\left(t_{i-1}\right) \mid b\left(t_{j}\right), t_{j}\right)\right) \varepsilon_{b, j},
\end{aligned}
$$


which can be rewritten as

$$
\begin{aligned}
\varepsilon_{b, i}= & -2 \sum_{j=1}^{i-1}\left(\left[1-F_{X\left(t_{i}\right)}\left(b\left(t_{i}\right) \mid a\left(t_{j}\right), t_{j}\right)\right]-\left[1-F_{X\left(t_{i-1}\right)}\left(b\left(t_{i-1}\right) \mid a\left(t_{j}\right), t_{j}\right)\right]\right) \varepsilon_{a, j} \\
& -2 \sum_{j=1}^{i-1}\left(\left[1-F_{X\left(t_{i}\right)}\left(b\left(t_{i}\right) \mid b\left(t_{j}\right), t_{j}\right)\right]-\left[1-F_{X\left(t_{i-1}\right)}\left(b\left(t_{i-1}\right) \mid b\left(t_{j}\right), t_{j}\right)\right]\right) \varepsilon_{b, j} \\
& +\frac{2}{h}\left(\delta_{1}\left(h, t_{i}\right)-\delta_{1}\left(h, t_{i-1}\right)\right), \\
\varepsilon_{a, i}= & -2 \sum_{j=1}^{i-1}\left(F_{X\left(t_{i}\right)}\left(a\left(t_{i}\right) \mid a\left(t_{j}\right), t_{j}\right)-F_{X\left(t_{i-1}\right)}\left(a\left(t_{i-1}\right) \mid a\left(t_{j}\right), t_{j}\right)\right) \varepsilon_{a, j} \\
& -2 \sum_{j=1}^{i-1}\left(F_{X\left(t_{i}\right)}\left(a\left(t_{i}\right) \mid b\left(t_{j}\right), t_{j}\right)-F_{X\left(t_{i-1}\right)}\left(a\left(t_{i-1}\right) \mid b\left(t_{j}\right), t_{j}\right)\right) \varepsilon_{b, j} \\
& +\frac{2}{h}\left(\delta_{2}\left(h, t_{i}\right)-\delta_{2}\left(h, t_{i-1}\right)\right) .
\end{aligned}
$$

Let us now consider the global error

$$
\xi_{i}=\left|\varepsilon_{a, i}\right|+\left|\varepsilon_{b, i}\right|
$$

When the hypotheses (16) and (17) are fulfilled,

$$
\left|\xi_{i}\right| \leq\left|\left(c_{1}+c_{2}\right) h\right| \sum_{j=1}^{i-1}\left|\xi_{j}\right|+\frac{2}{h}\left\|\delta_{1}\left(h, t_{i}\right)-\delta_{1}\left(h, t_{i-1}\right)|+| \delta_{2}\left(h, t_{i}\right)-\delta_{2}\left(h, t_{i-1}\right)\right\| .
$$

Observing that the Euler method errors are $\left|\delta_{1}(h, t)\right|=\left|\delta_{2}(h, t)\right|=O\left(h^{2}\right)$ and applying Theorem 7.1 of [19] we obtain $\left|\xi_{i}\right|=O\left(h^{2}\right)$ and, hence, the thesis.

Remark 7. A better result on the errors can be obtained by improving the integral discretization rule, i.e. using the midpoint formula instead of the Euler's method. Other integration rules can improve the order of the error, but strongly increase the computational complexity of the algorithm.

Remark 8. The two methods are equivalent in terms of computational time when the Laplace transform expression is a well-behaved function. Nevertheless, the generalization of the method for a time-dependent boundary $S(t)$ is possible only for the numerical method.

\section{Examples}

In this section we discuss a set of examples of interest for the applications, i.e. standard Brownian motion, geometric Brownian motion, and the Ornstein-Uhlenbeck process. We apply the algorithms of Section 4 for numerical evaluations. When the joint densities are known in closed form, we use them to illustrate the reliability of the algorithms.

\subsection{Standard Brownian motion}

Let us consider a standard Brownian motion with constant boundaries. It is a time and space homogeneous diffusion process; hence, we can rewrite its joint density functions (5) and (6) in 
(a)

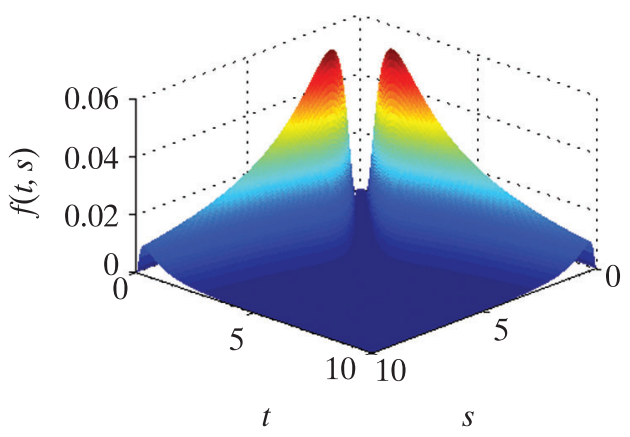

(c)

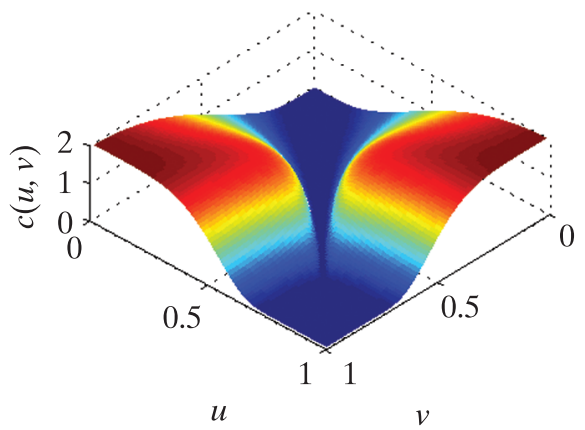

(b)

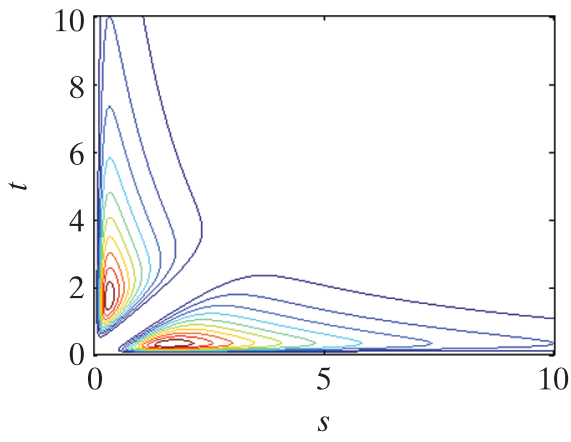

(d)

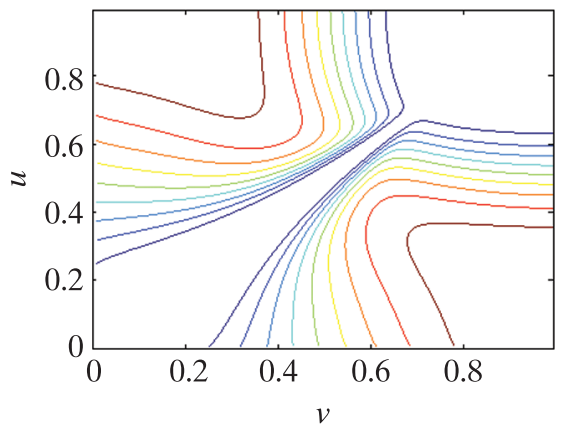

FIGURE 2: First hitting times of a Brownian motion through two constant boundaries $b=-a=1$. (a) The joint probability density function. (b) Contour lines of the joint probability density function. (c) The density of the copula. (d) Contour lines of the density of the copula. closed form as follows.

(i) If $x_{0}<a<b$ or $b<a<x_{0}$ then

$$
f_{T_{a} T_{b}}(t, s)= \begin{cases}0, & t \geq s \\ f_{T_{a}}(t \mid 0,0) f_{T_{b-a}}(s-t \mid 0,0), & t<s\end{cases}
$$

(ii) If $a<x_{0}<b$ then

$$
f_{T_{a} T_{b}}(t, s)= \begin{cases}f_{T_{b-a}}(s-t \mid 0,0) g_{a}(t \mid 0,0), & t<s \\ 0, & t=s \\ f_{T_{a-b}}(t-s \mid 0,0) g_{b}(s \mid 0,0), & t>s\end{cases}
$$

where $g_{a}\left(t \mid x_{0}, t_{0}\right)$ and $g_{b}\left(t \mid x_{0}, t_{0}\right)$ are given by (3) and $f_{T_{a}}(t \mid 0,0)$ is given by (4).

Figure 2 shows the joint probability density function of the first hitting times of a standard Brownian motion through two constant boundaries $b=-a=1$ and the corresponding copula together with the contour lines. Figure 3 illustrates the case of constant boundaries $a=-1$ and $b=1.5$ asymmetric with respect to $x_{0}$. The asymmetry of the boundary locations determines peaks of different heights. Note that the maximums of the joint density have inverted height in the corresponding copula. 
(a)

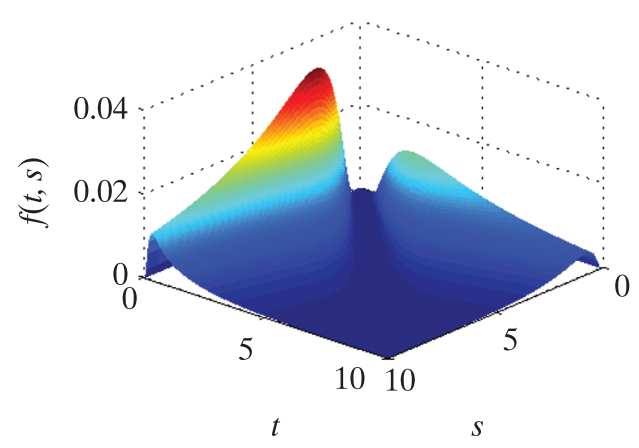

(c)

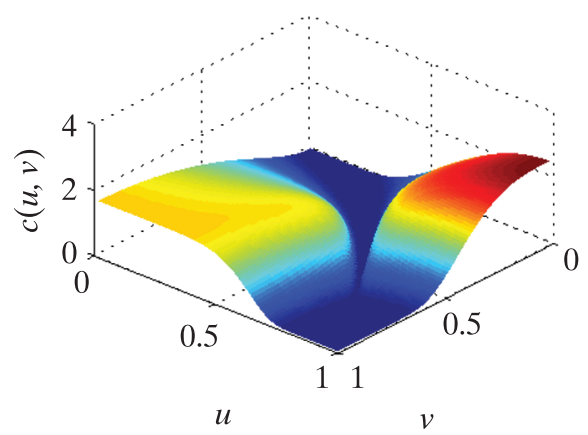

(b)

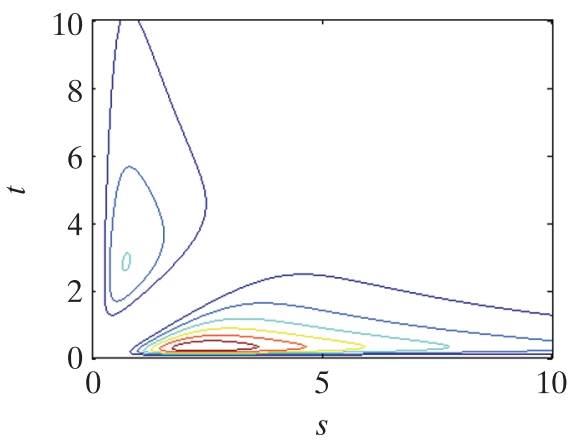

(d)

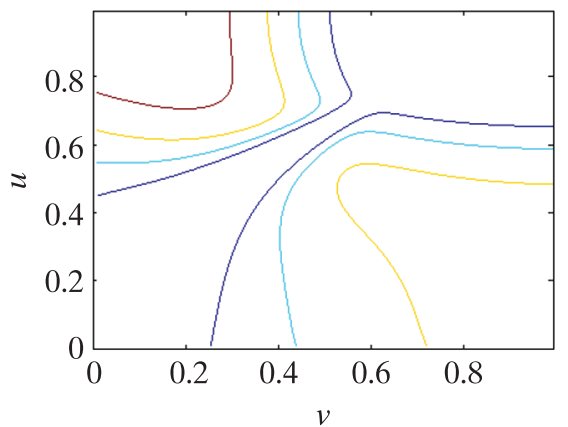

FIGURE 3: First hitting times of a Brownian motion through two constant boundaries $a=-1$ and $b=1.5$. (a) The joint probability density function. (b) Contour lines of the joint probability density function. (c) The density of the copula. (d) Contour lines of the density of the copula.

Furthermore, the Laplace transforms of $g_{a}^{\lambda}\left(x_{0}\right)$ and $g_{b}^{\lambda}\left(x_{0}\right)$ can be found in [5] or by applying Corollary 1.

Remark 9. The stability of the algorithms introduced in Section 4, already proved by Theorem 4 , is confirmed by the standard Brownian motion case where the probability density functions $g_{a}\left(t \mid x_{0}, t_{0}\right)$ and $g_{b}\left(t \mid x_{0}, t_{0}\right)$ are available in closed form. We apply the algorithms to the standard Brownian motion with constant boundaries $a=-1$ and $b=2$ with discretization step $h=0.01$, and we compare the results with the closed-form densities (3) for a series truncated at $N=10^{3}$ steps. The inversion of the Laplace transform with the Euler method gives a mean-square deviation

$$
\operatorname{MSE}_{a}=6.02 \times 10^{-19} \text { and } \operatorname{MSE}_{b}=9.83 \times 10^{-20} .
$$

The numerical algorithm gives a mean-square deviation

$$
\operatorname{MSE}_{a}=3.23 \times 10^{-6} \text { and } \operatorname{MSE}_{b}=5.11 \times 10^{-8} .
$$

It confirms the reliability of the new algorithm. The higher precision of the Laplace inversion with respect to the numerical method is determined by the simple expression of the involved Laplace transforms. However, we cannot infer an analogous property for the other diffusions. 
(a)

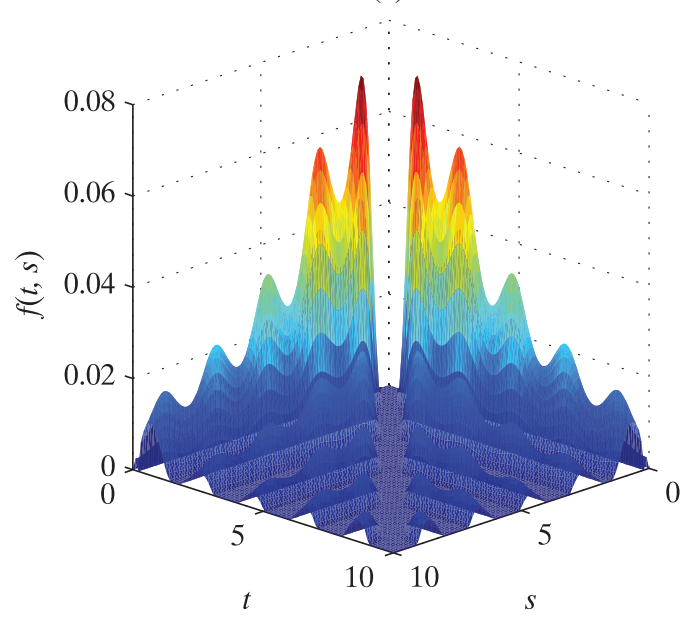

(b)

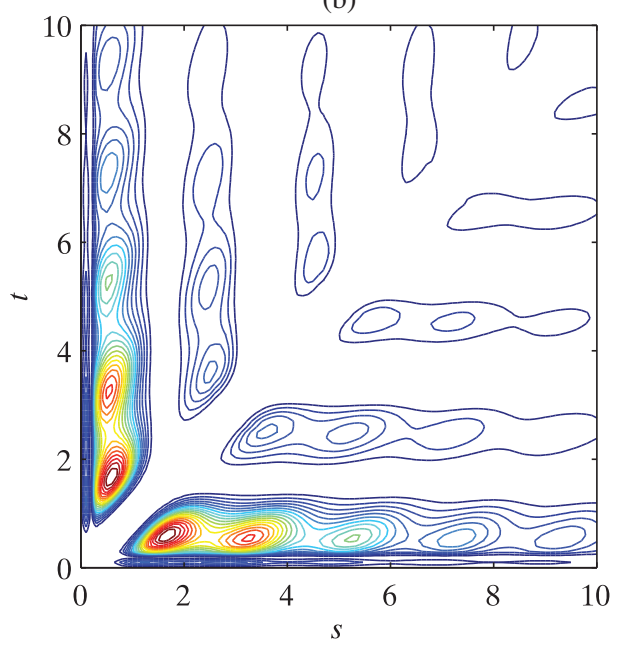

FiguRe 4: First hitting times of a Brownian motion through two time-dependent boundaries $b(t)=$ $1+0.1 \cos (\pi t)$ and $a(t)=-1+0.1 \cos (\pi t+\pi)$. (a) The joint probability density function. (b) Contour lines of the joint probability density function.

Remark 10. The extension of the above results to a Brownian motion with diffusion coefficient $\sigma \neq 1$ is straightforward. Indeed, a Brownian motion with diffusion coefficient $\sigma$ can be transformed into a standard Brownian motion via the space transformation $x=x^{\prime} / \sigma$, and the boundaries $a$ and $b$ become $a / \sigma$ and $b / \sigma$, respectively. When $\mu>0$ and $a(0)<x_{0}<b(0)$, we can determine $g_{b}\left(t \mid x_{0}, t_{0}\right)$. In this case the crossing of the boundary $a(t)$ is not a sure event and the study of $g_{a}\left(t \mid x_{0}, t_{0}\right)$ requires a suitable normalization. Similarly, the case in which $\mu<0$ and $a(0)<x_{0}<b(0)$ is analogous, interchanging the role of the two boundaries.

Remark 11. Denoting by $C_{T_{a}, T_{b}}^{\sigma}$ the copula of $\left(T_{a}, T_{b}\right)$ for a Brownian motion with diffusion coefficient $\sigma$ and by $C_{T_{a}, T_{b}}$ the copula in the case $\sigma=1$, recalling the transformation $x=x^{\prime} / \sigma$, the relationship $C_{T_{a}, T_{b}}^{\sigma}=C_{T_{a / \sigma}, T_{b / \sigma}}$ holds. Geometric Brownian motion can be obtained by a standard Brownian motion via the space transformation $x^{\prime}=\exp (\sigma x)$. The corresponding copula, $C_{T_{a}, T_{b}}^{\mathrm{GBM}}$, is related with the copula of the standard Brownian motion through

$$
C_{T_{a}, T_{b}}^{\mathrm{GBM}}=C_{T_{\ln a / \sigma}, T_{\ln a / \sigma}} .
$$

The more general transformation $x^{\prime}=\exp (\mu t+\sigma x)$ is not interesting from the point of view of the exit times from a strip because it corresponds to transforming the process into a Brownian motion with drift that does not have a sure crossing, as stated in Remark 10.

As a further example, we consider a standard Brownian motion with the boundaries

$$
b(t)=1+0.1 \cos (\pi t) \quad \text { and } \quad a(t)=-1+0.1 \cos (\pi t+\pi) .
$$

Since the boundaries are time dependent, Laplace transform inversions cannot be applied. Figure 4 shows the joint probability density function of the first hitting times and the corresponding contour lines obtained with the proposed numerical algorithm. 
(a)

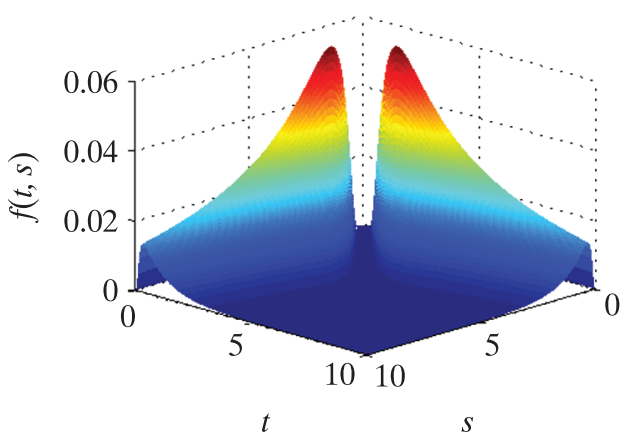

(c)

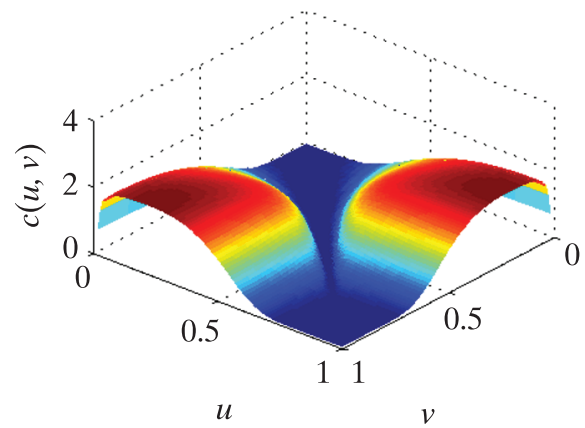

(b)

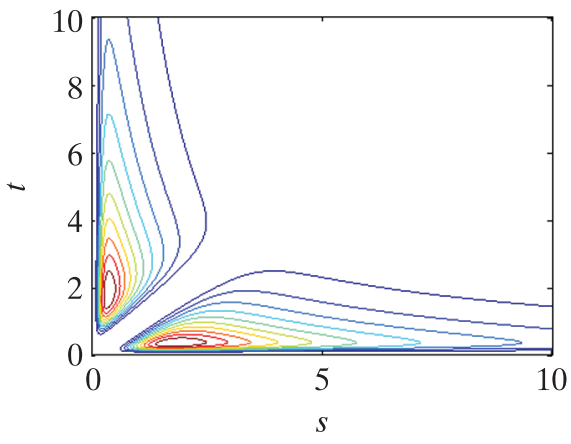

(d)

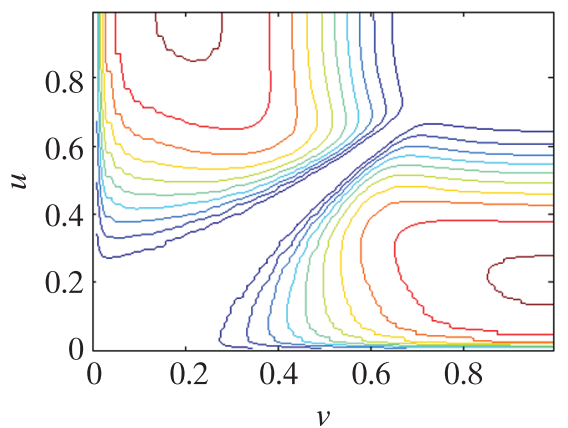

FIGURE 5: First hitting times of an Ornstein-Uhlenbeck process through two constant boundaries $b=$ $-a=1$. (a) The joint probability density function. (b) Contour lines of the joint probability density function. (c) The density of the copula. (d) Contour lines of the density of the copula.

\subsection{Ornstein-Uhlenbeck process}

Consider as a further example the Ornstein-Uhlenbeck process, described by the stochastic differential equation

$$
\mathrm{d} X(t)=\left(-\frac{X(t)}{\theta}+\mu\right) \mathrm{d} t+\sigma \mathrm{d} W_{t}, \quad X\left(t_{0}\right)=x_{0}
$$

For this process, representations and numerical methods are available and can be used to evaluate the first hitting time probability density function $f_{T_{a}}\left(t \mid x_{0}, t_{0}\right)$ (see [3], [6], and [18]). On the other hand, the density $g_{a}\left(t \mid x_{0}, t_{0}\right)$ is not known in closed form. Here we have applied classical numerical algorithms (cf. [6]) to evaluate the first hitting time probability density function and the algorithms of Section 4 to compute the second. Figure 5 shows the joint probability density function and the corresponding copula of the first hitting times of an Ornstein-Uhlenbeck process with parameters $\theta=10, \mu=0, \sigma=1$, and $x_{0}=0$ through two constant boundaries $b=-a=1$. Figure 6 illustrates the case of asymmetric with respect to $x_{0}$ constant boundaries $a=-1$ and $b=1.5$. Note that $x_{0}$ represents the symmetry axis of the Ornstein-Uhlenbeck process. The height of the peaks of the joint density and of the copula behaves as the Brownian motion case. 
(a)

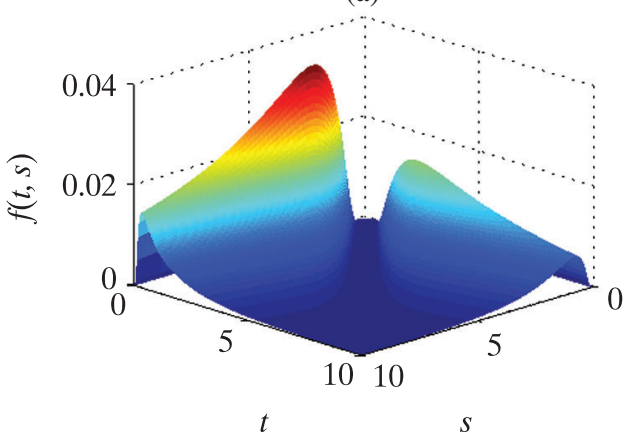

(c)

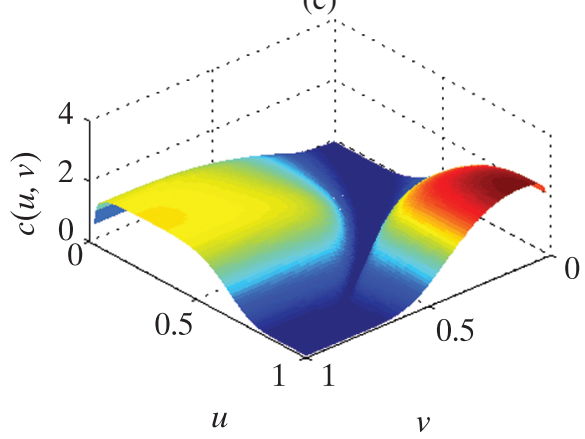

(b)

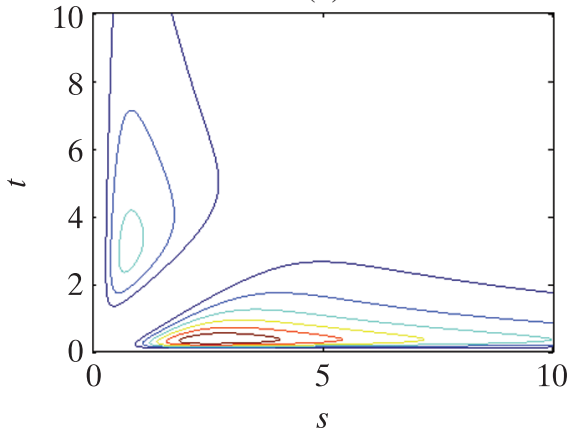

(d)

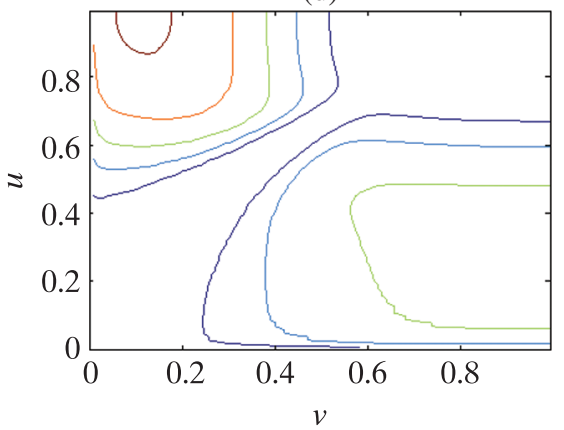

FIGURE 6: First hitting times of an Ornstein-Uhlenbeck process through two constant boundaries $a=-1$ and $b=1.5$. (a) The joint probability density function. (b) Contour lines of the joint probability density function. (c) The density of the copula. (d) Contour lines of the density of the copula.

The Laplace transforms $g_{a}^{\lambda}\left(x_{0}\right)$ and $g_{b}^{\lambda}\left(x_{0}\right)$ for the Ornstein-Uhlenbeck process can be found in [5]. However, the presence of the parabolic cylinder functions in their expression discourage their numerical inversion.

\section{Acknowledgements}

We are grateful to an anonymous referee for interesting and constructive comments that helped to improve the paper. This work was supported in part by the 2012 University of Torino grant 'Stochastic Processes and their Applications' and by project A.M.A.L.F.I.-Advanced Methodologies for the Analysis and management of the Future Internet (Università di Torino/ Compagnia di San Paolo).

\section{References}

[1] AвAte, J. ANd Whitt, W. (1995). Numerical inversion of Laplace transforms of probability distributions. ORSA J. Comput. 7, 36-43.

[2] Albano, G. And Giorno, V. (2006). A stochastic model in tumor growth. J. Theoret. Biol. 242, 329-336.

[3] Alili, L., Patie, P. And Pedersen, J. L. (2005). Representations of the first hitting time density of an OrnsteinUhlenbeck process. Stoch. Models 21, 967-980.

[4] Benedetto, E., Sacerdote, L. and Zucca, C. (2013). A first passage problem for a bivariate diffusion process: numerical solution with an application to neuroscience when the process is Gauss-Markov. J. Comput. Appl. Math. 242, 41-52. 
[5] Borodin, A. N. And Salminen, P. (2002). Handbook of Brownian Motion-Facts and Formulae, 2nd edn. Birkhäuser, Basel.

[6] Buonocore, A., Nobile, A. G. And Ricciardi, L. M. (1987). A new integral equation for the evaluation of first-passage-time probability densities. Adv. Appl. Prob. 19, 784-800.

[7] Buonocore, A., Giorno, V., Nobile, A. G. And Ricciardi, L. M. (1990). On the two-boundary first-crossingtime problem for diffusion processes. J. Appl. Prob. 27, 102-114.

[8] Capocelli, R. M. and Ricciardi, L. M. (1976). On the transformation of diffusion processes into the Feller process. Math. Biosci. 29, 219-234.

[9] Davydov D. AND Linetsky, V. (2003). Pricing options on scalar diffusions: an eigenfunction expansion approach. Operat. Res. 51, 185-209.

[10] Di Crescenzo, A. G., Giorno, V., Nobile, A. G. and Ricciardi, L. M. (1995). On a symmetry-based constructive approach to probability densities for two-dimensional diffusion processes. J. Appl. Prob. 32, 316336.

[11] Galleani, L., Sacerdote, L., Tavella, P. and Zucca, C. (2003). A mathematical model for the atomic clock error. Metrologia 40, S257-S264.

[12] Giorno, V., Nobile, A. G. And Ricciardi, L. M. (2011). On the densities of certain bounded diffusion processes. Ric. Mat. 60, 89-124.

[13] Giorno, V., Nobile, A. G., Ricciardi, L. M. and Sacerdote, L. (1986). Some remarks on the Rayleigh process. J. Appl. Prob. 23, 398-408.

[14] Giraudo, M. T. and Sacerdote, L. (1999). An improved technique for the simulation of first passage times for diffusion processes. Commun. Statist. Simul. Comput. 28, 1135-1163.

[15] Giraudo, M. T., Sacerdote, L. and Zucca, C. (2001). A Monte Carlo method for the simulation of first passage times of diffusion processes. Meth. Comp. Appl. Prob. 3, 215-231.

[16] Itô, K. And McKean, H. P., JR. (1974). Diffusion Processes and Their Sample Paths. Springer, Berlin.

[17] Lapidus, L. And Pinder, G. F. (1999). Numerical Solution of Partial Differential Equations in Science and Engineering. John Wiley, New York.

[18] Linetsky, V. (2004). Computing hitting time densities for CIR and OU diffusions: applications to mean-reverting models. J. Comput. Finance 7, 1-22.

[19] LinZ, P. (1985). Analytical and Numerical Methods for Volterra Equations (SIAM Studies Appl. Math. 7). SIAM, Philadelphia, PA.

[20] Nelsen, R. B. (1999). An Introduction to Copulas (Lecture Notes Statist. 139). Springer, New York.

[21] Novikov, A., Frishling, V. And KordzakHIA, N. (1999). Approximations of boundary crossing probabilities for a Brownian motion. J. Appl. Prob. 36, 1019-1030.

[22] Orsingher, E. And Beghin, L. (2006). Probabilità e Modelli Aleatori. Aracne, Roma.

[23] Panfilo, G., Tavella, P. and Zucca, C. (2004). How long does a clock error remain inside two threshold barriers? An evaluation by means of stochastic processes. In Proc. Europ. Freq. Time Forum, Guilford, pp. 110115.

[24] Peskir, G. (2002). Limit at zero of the Brownian first-passage density. Prob. Theory Relat. Fields 124, $100-111$.

[25] Ricciardi, L. M. (1976). On the transformation of diffusion processes into the Wiener process. J. Math. Anal. Appl. 54, 185-199.

[26] Ricciardi, L. M. (1977). Diffusion Processes and Related Topics in Biology (Lecture Notes Biomath. 14). Springer, Berlin.

[27] Ricciardi, L. M. and Sacerdote, L. (1987). On the probability densities of an Ornstein-Uhlenbeck process with a reflecting boundary. J. Appl. Prob. 24, 355-369.

[28] Ricciardi, L. M. and Sato, S. (1990). Diffusion processes and first-passage-time problems. In Lectures in Applied Mathematics and Informatics, Manchester University Press, pp. 206-285.

[29] Ricciardi, L. M., SACERdote, L. AND SATo, S. (1984). On an integral equation for first-passage-time probability densities. J. Appl. Prob. 21, 302-314.

[30] Ricciardi, L. M., Di Crescenzo, A., Giorno, V. and Nobile, A. G. (1999). An outline of theoretical and algorithmic approaches to first passage time problems with applications to biological modeling. Math. Japon. 50, 247-322.

[31] Sacerdote, L. and Giraudo, M. T. (2013). Stochastic integrate and fire models: a review on mathematical methods and their applications. In Stochastic Biomathematical Models (Lecture Notes Math. 2058), pp. 99-148.

[32] Sмith, G. D. (1978). Numerical Solution of Partial Differential Equations. Finite Difference Methods, 2 nd edn. Oxford University Press.

[33] Smith, P. L. (2000). Stochastic dynamic models of response time and accuracy: a foundational primer. J. Math. Psych. 44, 408-463.

[34] VAN LOAN, C. (1992). Computational Frameworks for the Fast Fourier Transform. SIAM, Philadelphia, PA.

[35] Zucca, C. ANd Sacerdote, L. (2009). On the inverse first-passage-time problem for a Wiener process. Ann. Appl. Prob. 19, 1319-1346. 